techniques, pyrolytic derivatisation gas-liquid chromatography with mass spectrometry (GC-MS) detection, and several other forms of GC-MS. Although none of these techniques is a routine diagnostic method, all are potentially useful. I was particularly interested in the chapter on infrared spectrometry, as it described a promising technology at an advanced stage of development. Raman spectrometry offers an exciting new non-destructive method for specific analysis of many important cell components in situ. The various GC-MS techniques have clear potential applications in chemical analysis of small samples of cells and, possibly, direct detection of organisms in clinical samples. All the techniques described require minimal specimen preparation and dispense with, or minimise, the incubation phase which is an inherent limitation of classical microbiological techniques. Readers who are willing to accept that microbiology must move out of the petri dish will find this a particularly interesting book. It shows clearly that other disciplines have developed approaches that could be adapted to our needs and revolutionise microbiology. Be prepared to wrestle with some chemistry, mathematics and complex physical principles, but it is a worthwhile effort, revealing a large slice of state of the art technology that we could usefully assimilate, given enterprise and a more inter-disciplinary approach to microbiology.

J. T. MAGEE

\section{Review of Parasitic Zoonoses}

S. C. Parija. 1990. ISBN 81-85386-21-8. AITBS Publishers, Delhi. Pp. 463. Rs 225.

The contributors to this review are experts from medical and veterinary institutions of India (the only exception being the chapter on toxoplasmosis by a veterinary microbiologist from Maryland, USA). The text is presented in a very readable and attractive manner. Salient points on life cycle, prevalance of the disease in India, classification etc, have been highlighted with the help of boxes, line diagrams and tables.

The review is intended for both undergraduate and postgraduate students of medical, veterinary and allied sciences and can be commended to these groups, particularly in India and other tropical countries. On a wider perspective, in an ever shrinking world, it will be handy in clinical laboratories anywhere around the globe where, in view of the rarity of a particular condition, expertise might not be readily available.

The only omission I feel is the lack of discussion of parasitic zoonoses associated with the Acquired Immune Deficiency Syndrome-perhaps in a separate chapter. Over the past decade this condition has led to better understanding of the pathogenicity and potential severity of parasitic diseases, and has focused attention on the need for better diagnostic and therapeutic facilities.

This book will serve as a handy reference for the next time I need information on an uncommon parasitic zoonosis.

A. GHOSE

\section{Atlas of Medical Helminthology and Protozoology}

Revised by G. O. Cowan. 1991. ISBN 0443040931. Churchill Livingstone, Edinburgh. Pp. 78. Illustrations 121. $£ 30$.00.

Parasitic disease is of enormous global importance: well over a million children die of malaria each year, and hundreds of millions of people suffer from other major parasitic infections, including filariasis, schistosomiasis, leishmaniasis and trypanosomiasis; infection with intestinal helminths is even more prevalent, affecting billions of people, predominantly in the developing world.

Traditionally, medical microbiologists have been inclined to leave the study of protozoa and helminths to the experts of the Tropical Schools and Hospitals. This was always a dangerous philosophy, since many parasites are cosmopolitan in distribution, but it is now completely untenable with the speed of modern travel which ensures that 'tropical' diseases are encountered in every corner of the world. Parasitic diseases also feature prominently among the opportunist infections to which AIDS patients are prone and it is more important than ever that medical microbiologists should take the subject seriously.

Little has changed in the parasitological world since the publication of the first edition of Jeffrey and Leach's popular atlas in 1966. The new, third edition, still in its spiral-bound format, but larger and slightly unwieldy in its dimensions, has been revised by Colonel Cowan by the inclusion of "new" parasites, like Cryptosporidium spp. and by updating the nomenclature. The opportunity has been taken for some judicious pruning, including the omission of some of the less important parasites and, unusually among textbooks, the third edition has fewer pages than the first! Once again, the life cycles are presented in clear, succinct form, making use of R. H. Leach's original drawings. However, much of the original text, which was in note form, has been expanded into true text, together with maps illustrating the geographical distribution, and this is a considerable improvement. The new index, though short, also adds to the usefulness of the book.

The purpose of this book, as stated in the Preface to the first edition, is primarily to provide a teaching aid in parasitology. In this it succeeds admirably. It packs a great deal of useful information into a small space by its diagrammatic approach and the salient features of the life cycles are easily assimilated. However, as a bench book it is less successful: the drawings, though accurate do not conform to the reality one sees down the microscope. The representations of Pneumocystis carinii, Leishmania spp. and many other parasites would be of little help to those unfamiliar with the real thing. In this respect, a true atlas with actual photomicrographs, such as Peters and Gilles' Colour atlas of tropical medicine and parasitology is far superior. Other shortcomings are the lack of guidance on further reading and the price, which seems excessive at nearly $40 \mathrm{p} /$ page.

"The protozoon and the helminth", as the introduction to this new edition of Jeffrey and Leach's atlas reminds us, quoting the words of Sir Patrick Manson in 1899, "are in the ascendant". Nothing has changed, and those wishing to know more of these fascinating creatures will find the essentials of the subject laid out with great clarity in this book.

D. Greenwood and G. Payne 